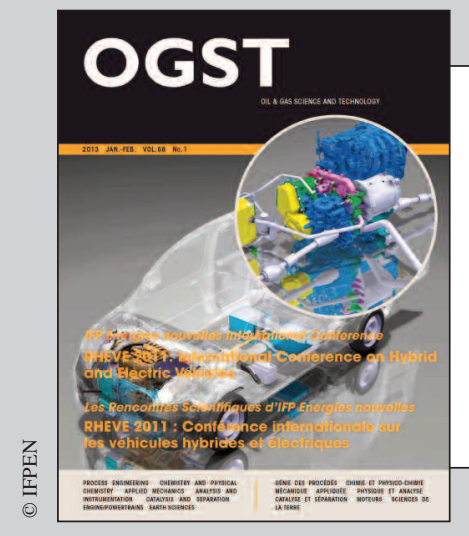

Dossier

This paper is a part of the hereunder thematic dossier published in OGST Journal, Vol. 68, No. 1, pp. 3-178 and available online here

Cet article fait partie du dossier thématique ci-dessous publié dans la revue OGST, Vol. 68, n 1 , pp. 3-178 et téléchargeable ici

DOSSIER Edited by/Sous la direction de : A. Sciarretta, F. Badin et J. Bernard RHEVE 2011 : International Conference on Hybrid and Electric Vehicles RHEVE 2011 : Conférence internationale sur les véhicules hybrides et électriques

Oil \& Gas Science and Technology - Rev. IFP Energies nouvelles, Vol. 68 (2013), No. 1, pp. 3-178

Copyright (C) 2013, IFP Energies nouvelles

\section{$3>$ Editorial}

$13>$ Analysis and Experimental Implementation of a Heuristic Strategy for Onboard Energy Management of a Hybrid Solar Vehicle

Analyse et expérimentation d'une stratégie heuristique pour la gestion d'énergie à bord d'un véhicule hybride solaire

G. Coraggio, C. Pisanti, G. Rizzo and M. Sorrentino

23 > Open Issues in Supervisory Control of Hybrid Electric Vehicles: A Unified Approach Using Optimal Control Methods

Questions ouvertes sur la supervision énergétique des véhicules hybrides électriques : une approche unifiée par la théorie de la commande optimale

L. Serrao, A. Sciarretta, 0. Grondin, A. Chasse, Y. Creff, D. Di Domenico, P. Pognant-Gros, C. Querel and L. Thibault

35 > Optimization of Hybrid Power Trains by Mechanistic System Simulations

Optimisation de groupes motopropulseurs électriques hybrides par simulation du système mécanique

T. Katrašnik and J.C. Wurzenberger

51 > A Phenomenological Heat Transfer Model of SI Engines - Application to the Simulation of a Full-Hybrid Vehicle

Un modèle phénoménologique de transfert thermique au sein de moteurs à allumage commandé - Application à la simulation d'un véhicule full-hybride

R. Dubouil, J.-F. Hetet and A. Maiboom

$65>$ Battery Electric Vehicle (BEV) or Range Extended Electric Vehicle (REEV)? - Deciding Between Different Alternative Drives Based on Measured Individual Operational Profiles

Véhicule électrique à batteries (BEV) ou véhicule électrique à prolongateur d'autonomie (REEV) ? - Choisir entre différents entrânements alternatifs sur la base de profils opérationnels individuels mesurés

S. Marker, B. Rippel, P. Waldowski, A. Schulz and V. Schindler

$79>$ Assessment by Simulation of Benefi ts of New HEV Powertrain Configurations

Évaluation par simulation des bénéfi ces de nouvelles chaînes de traction hybrides

N. Kim and A. Rousseau
95 > Dual Mode Vehicle with In-Wheel Motor: Regenerative Braking Optimization

Véhicule bi-mode avec moteurs roues : optimisation du freinage récupératif

G. Le Solliec, A. Chasse, J. Van-Frank and D. Walser

109 > Engine Downsizing and Electric Hybridization Under Consideration of Cost and Drivability

Réduction de taille moteur et hybridation électrique avec considérations de coût et de performance de conduite

S. Ebbesen, P. Elbert and L. Guzzella

117 > Representative Midwestern US Cycles: Synthesis and Applications Cycles représentatifs du Middle West américain : synthèse et applications

T.-K. Lee and Z.S. Filipi

127 > A Review of Approaches for the Design of Li-lon BMS Estimation Functions

Revue de différentes approches pour l'estimation de l'état de charge de batteries Li-ion

D. Di Domenico, Y. Creff, E. Prada, P. Duchêne, J. Bernard and V. Sauvant-Moynot

137 > Experimental Assessment of Battery Cycle Life Within the SIMSTOCK Research Program

Évaluation expérimentale de la durée de vie de la batterie dans le programme de recherche SIMSTOCK

P. Gyan, P. Aubret, J. Hafsaoui, F. Sellier, S. Bourlot, S. Zinola and F. Badin

$149>$ Smart Battery Thermal Management for PHEV Efficiency Une gestion avancée de la thermique de la batterie basse tension de traction pour optimiser l'efficacité d'un véhicule hybride électrique rechargeable

L. Lefebvre

$165>$ Parameterization and Observability Analysis of Scalable Battery Clusters for Onboard Thermal Management

Paramétrage et analyse d'observabilité de clusters de batteries de taille variable pour une gestion thermique embarquée

Xinfan Lin, Huan Fu, Hector E. Perez, Jason B. Siege, Anna G. Stefanopoulou, Yi Ding and Matthew P. Castanier 


\title{
Representative Midwestern US Cycles: Synthesis and Applications
}

\author{
T.-K. Lee ${ }^{1 *}$ and Z.S. Filipi ${ }^{2}$ \\ 1 Department of Mechanical Engineering, University of Michigan, Ann Arbor, MI 48109 - USA \\ 2 Department of Automotive Engineering, Clemson University, Greenville, SC 29607-5257 - USA \\ e-mail: kteel@umich.edu - zfilipi@clemson.edu \\ * Corresponding author
}

\begin{abstract}
Résumé - Cycles représentatifs du Middle West américain : synthèse et applications - Cet article propose un ensemble de cycles de conduite représentatifs du monde réel dans le Middle West américain, aptes à reproduire la dépendance des modes de conduite à la distance parcourue. Des analyses récentes de la conduite aux Etats-Unis montrent que la plupart des cycles de certification mènent à une sousestimation de la consommation d'énergie par mile parcouru par rapport aux habitudes de conduite.

La conduite dans le monde réel est un mix de conduite locale et de conduite sur autoroutes. De plus, les habitudes de conduite montrent une forte dépendance à la distance parcourue. Pour couvrir la vaste gamme de distances parcourues dans le monde réel, cinq cycles synthétiques ont été générés, allant de 4,78 miles à 40,71 miles, conformément à la répartition des distances parcourues dans le monde réel. Chaque cycle individuel est construit par un processus stochastique utilisant les informations de conduite extraites de données de trajets dans le Middle West américain. Lors de la construction de l'ensemble des cycles, les critères statistiques de validation de la représentativité des cycles sont traités afin de pouvoir reproduire la dépendance à la distance et d'éliminer les variations aléatoires. Les cycles synthétisés sont ensuite utilisés pour des études de conception et de contrôle de véhicules hybrides électriques ou électriques rechargeables afin d'évaluer l'impact des véhicules électrifiés sur le réseau.
\end{abstract}

\begin{abstract}
Representative Midwestern US Cycles: Synthesis and Applications - This paper proposed a set of representative real-world driving cycles in Midwestern US, which are capable of capturing the dependence of driving patterns on driving distance. Recent analyses of the real-world driving in USA show that most of certification cycles lead to underestimation of energy consumption per mile compared to the naturalistic driving patterns. Real-world driving is a mix of local driving and highway driving. Furthermore, the driving patterns show high dependency on the driving distance. To cover the wide range of real-world driving distances, five synthetic cycles are generated ranging from 4.78 miles to 40.71 miles following the real-world driving distance distribution. Each individual cycle is constructed by a stochastic process using the extracted driving information from the naturalistic trip data in the Midwestern US. While constructing the cycle set, the statistical criteria for validating the cycle representativeness are processed to capture the clear distance dependency and remove random variations. The synthesized cycles are subsequently used for Plug-in Hybrid Electric Vehicle (PHEVs) or Hybrid Electric Vehicle (HEVs) design and control studies for the assessment of the impact of electrified vehicles on the grid.
\end{abstract}




\section{INTRODUCTION}

Strict regulations on fuel economy of vehicles and greenhouse gas emission reduction put strong emphasis on the development of Hybrid Electric Vehicles (HEVs) and Plug-in Hybrid Electric Vehicles (PHEVs). The regulations mandate the $120 \mathrm{~g} \mathrm{CO}_{2} / \mathrm{km}$ in the European Union and the $35.5 \mathrm{mpg}$ (mile par gallon) fleet fuel economy by 2016 in US. Hybrid propulsion system allows exceptional fuel economy improvements through flexible use of multiple power sources on board of a vehicle, engine shut-downs and recuperation of braking energy. Hence, the usage of stored electricity for vehicle propulsion in PHEVs presents promising ways for reducing the dependency on petroleum in the transportation sector and for facilitating future growth of renewable energy sources on the power grid.

Design optimization and supervisory control strategy are key elements in developing HEVs and PHEVs to obtain the full benefit of vehicle electrification. Vehicle design should be determined to satisfy the driving performance under real-world driving and vehicle control strategy should be developed to maximize the vehicle hardware potential. Since overall vehicle performance specification and hardware design are determined under applied driving cycles, realworld driving patterns must be considered in the very initial development stage to achieve the better fuel economy and performance.

Until present, certification driving cycles have been predominantly used to assess vehicle performance and fuel economy (Carlson et al., 2009; Duoba et al. 2009). The cycles include UDDS (Urban Dynamometer Driving Schedule) (Kruse and Huls, 1973) and HWFET (Highway Fuel Economy Test) cycles. New European Driving Cycle (NEDC) is typically used by European researchers. However, measured naturalistic driving cycles show a wide spectrums of driving patterns. Naturalistic cycles tend to be more aggressive than certification cycles (Patil et al., 2009). The discrepancy between certification cycles and real-world driving cycles tends to become larger with increased trip length. Thus, driving cycles play a critical role to obtain more realistic and reliable vehicle analysis and optimization results (Fellah et al., 2009; Kwon et al., 2008). In case of PHEVs, driving cycles are even more important since electric driving ranges, such as "All Electric Range (AER)" or "Mostly Electric Range (MER)", are directly influenced by driving patterns. Thus, capturing features of realistic driving patterns with a set of representative real-world driving cycles is indispensable for in-depth analysis of vehicle design and control strategy development.

Real-world driving patterns have strong dependency on trip distance. For instance, vehicles are not normally driven in low-speed city conditions for 30 or 40 miles and long commutes typically involve a portion of higher speed freeway driving. Thus, the information about real-world driving and its integration into vehicle analysis is indispensable for the large scale life-cycle analysis of energy use in transportation and the impact of the power-generation mix on the greenhouse gas emission. To represent real-world driving patterns in Europe, ARTEMIS European driving cycles were developed (André, 2004). The ARTEMIS cycles were composed by assembling adequately classified segments out of the database collected during actual driving of European cars and by subsequent representativeness validation process. An alternative approach that utilizes Markov chain and Transition Probability Matrices (TPMs) augmented by statistical analysis for validating representativeness was recently proposed by Lee and Filipi (2010, 2011a).

This paper proposes a procedure to synthesize a set of representative real-world driving cycles and its applications. The proposed cycles are capable of capturing the dependency of driving patterns on driving distance based on the methodology. The trip distance dependency is captured from the extracted driving pattern information in each divided segment on daily driving distance distribution. To synthesize driving cycles, Markov chain is used with its capability of representing naturalistic driving information in a compact form as proposed in Lee and Filipi (2011a). The proposed methodology has a unique flexibility in constructing arbitrary distance cycles with desired driving characteristics. Furthermore, the resulting synthetic cycles are general and independent of vehicle types and vehicle control strategy, since the proposed approach uses only velocity and acceleration data, i.e. it does not include vehicle related information or subjective parameters while synthesizing schedules.

In the present paper, real-world driving data are analysed and driving distance distribution is modeled first. Then, driving cycle synthesis procedure is described. Midwestern US driving cycles, typical of urban/suburban driving in a Midwestern US region are proposed and analysed. A response surface approach is then introduced to assess the impact of a large fleet PHEV on the grid in the application section. Finally, this paper ends up with conclusions.

\section{DRIVING DATA IN MIDWESTERN US}

Real-world driving data in Southeast Michigan collected by the University of Michigan Transportation Research Institute (UMTRI) by Field Operational Test (FOT) (LeBlanc et al., 2006) are used to analyze naturalistic driving patterns in the Midwest US area. Total 830 days 4409 trips were used for the analysis of real-world driving patterns. The data includes driving information sufficient for representing real-world driving patterns with respect to trip distance. Daily driving distance distribution is shown in Figure 1. Daily driving distance is a summation of trip lengths during one day.

Driving distance distribution is regressed to find a smoothed Probability Density Function (pdf) with the purpose of dividing driving data into several segments with the same 
probability depending on driving distance. Since the distribution is skewed as show in Figure 1, a Chi-square distribution $\left(\chi^{2}\right.$-distribution) is used for the regression model. The Chi-square distribution is expressed as:

$$
P\left(x_{n}\right)=\frac{x_{n}{ }^{(v-2) / 2} e^{-x_{n} / 2}}{2^{v / 2} \Gamma(v / 2)}
$$

where $\Gamma($.$) is the Gamma function defined as:$

$$
\Gamma(z)=\int_{0}^{\infty} t^{z-1} e^{-1} d t
$$

$x_{n}$ is the normalized driving distances defined as $x / \Delta d, x$ is the departure time, $\Delta d$ is the reference discretized step of the driving distance corresponding to the histogram and $v$ is determined to minimize the root mean square (rms) error of the response variable. The regressed function shows a smoothed curve fit to the raw data distribution. The probability distribution satisfies:

$$
\int P\left(x_{n}\right) d x_{n}=1
$$

Figure 2 shows the regressed pdf and the Cumulative Distribution Function (cdf) of one-day driving distances. Driving distance dependent driving patterns are captured from the driving cycle data divided into ten segments having the same probability on the cdf. Representative driving distance in each segment is selected as the mean value of the segment range. The selected one-way trip distances range from 4.78 to 40.71 miles.

While synthesizing cycles, driving pattern information is extracted from each segment. Initially, ten independent cycles are constructed. Then, five cycles, marked as solid circles, are selected to be members of a representative set, capable of capturing driving features as a function of trip length. The synthesis procedure is presented in Section 2 and the resulting cycles are proposed in Section 3.

\section{CYCLE SYNTHESIS PROCEDURE}

Generalized real-world driving patterns include both local trips and free-way trips. Driving patterns are different with respect to driving distances. Thus, the driving distance based categorization (Lee and Filipi, 2011a) is used to synthesize Southeast Michigan Urban/Suburban driving cycles in this paper.

The overall procedure is illustrated in Figure 3. A stochastic process combined with subsequent assessment procedure can construct driving cycles with verified representativeness (Lee and Filipi, 2010). Initially, naturalistic driving cycles for the extraction of real-world driving information are selected within each concerning segment. Driving information is extracted in a form of velocity and acceleration matrices (see Fig. 4). The matrices relate current velocity and acceleration to future information. Every current state is mapped to the states in the next time step (i.e., future time step) one-to-one. Markov chain uses the information to synthesize the cycles.

In this paper, a discrete-time Markov chain is used and it is a sequence of random variables $X_{1}, X_{2}, X_{3}$, etc. with the Markov property are expressed as:

$$
\begin{aligned}
& P\left(X_{n+1}=x_{n+1} \mid X_{1}=x_{1}, X_{2}=x_{2}, \ldots, X_{n}=x_{n}\right) \\
& =P\left(X_{n+1}=x_{n+1} \mid X_{n}=x_{n}\right)
\end{aligned}
$$

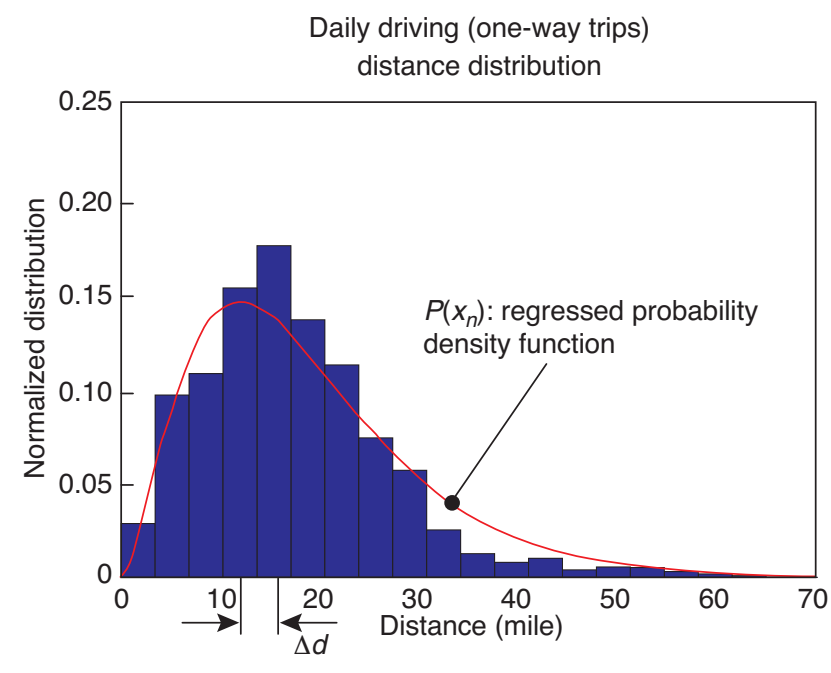

Figure 1

Daily driving distance distribution.
Daily driving (one-way trips) distance distribution

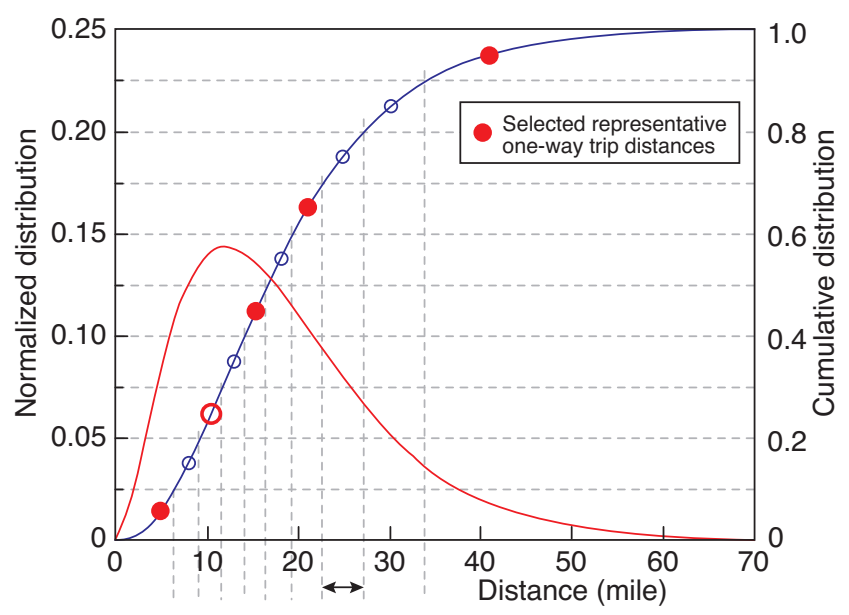

Figure 2

Statistical distribution of daily driving distances: probability density function, cumulative density function and selected representative one-way trip distances. 


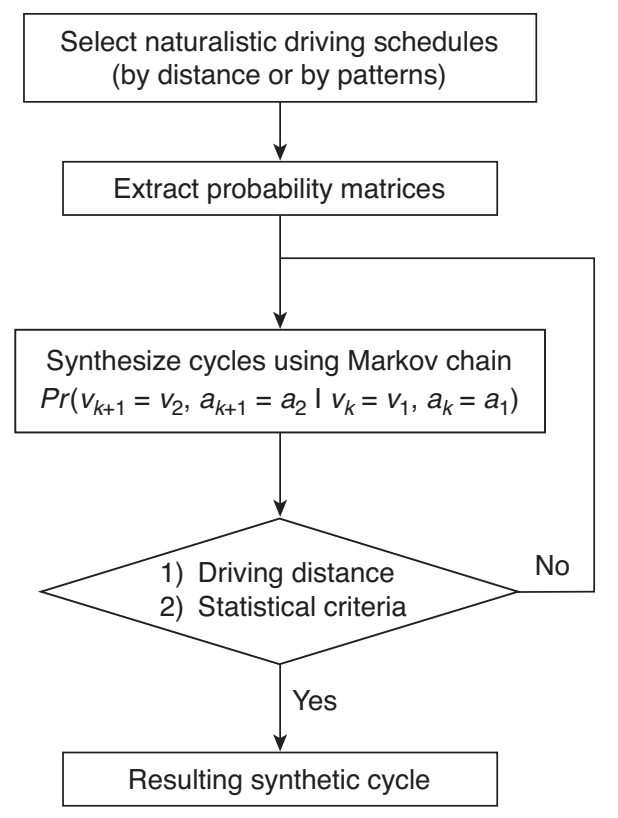

Figure 3

Naturalistic driving cycle synthesis procedure using Markov chain and statistical criteria.
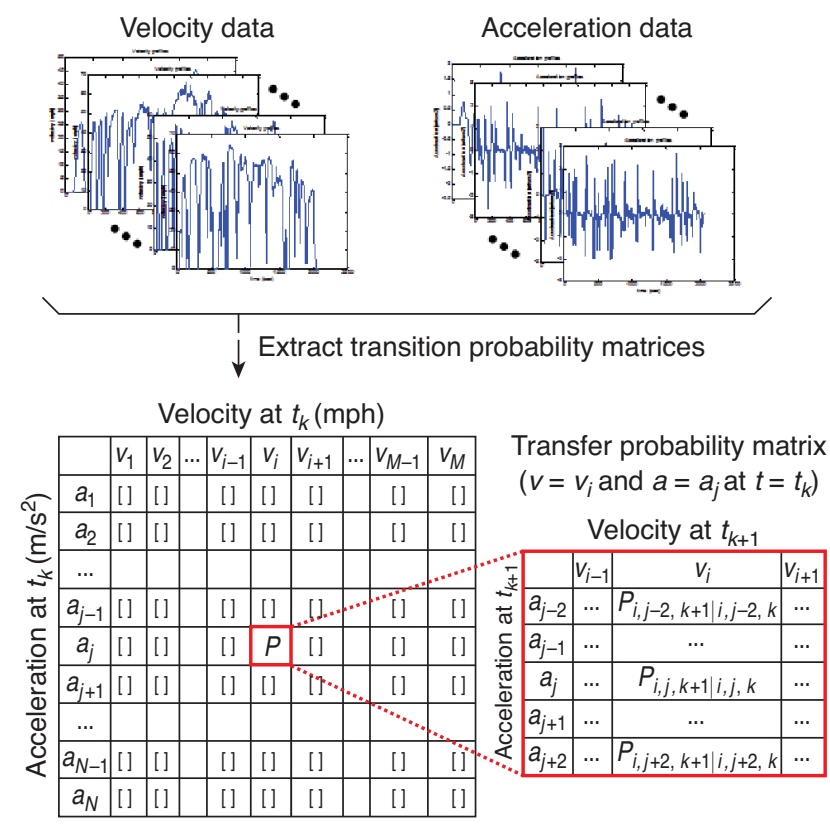

Figure 4

Illustration of the procedure to extract Transition Probability Matrix (TPM) from real-world driving data.
The set of possible values that the random variables $X_{n}$ can take is the state space of the chain. The conditional probabilities $p_{i j}=P\left(X_{n+1}=j \mid X_{n}=i\right)$ are transition probabilities. The probability used in the synthesis procedure is timeindependent (or time-homogeneous). The sum of all probabilities leaving a state must satisfy:

$$
\sum_{j} p_{i j}=\sum_{j} P\left(X_{n+1}=j \mid X_{n}=i\right)=1
$$

To satisfy the Markov property in Equation (4) that represents future states depend only on the present states, an adequate number of states should be chosen. The required states are selected by investigating the simplified vehicle dynamics equation. The vehicle dynamics can be expressed by velocity and acceleration and they are chosen as the states for the Markov chain. The TPM is then generated in the form of a two dimensional matrix with velocity and acceleration at current time $t_{k}$. The velocity and acceleration are discretized with the number of $M$ and $N$, respectively. The number of events at the next time step $t_{k+1}$ is counted at each current velocity $v_{k}$ and current acceleration $a_{k}$ at the present time step $t_{k}$, then divided by the total number of event to construct the probability matrix. Then, the conditional probability is expressed as:

$$
P_{i, j, k+1 \mid p, q, k}=P\left(v_{k+1}=v_{i}, a_{k+1}=a_{j} \mid v_{k}=v_{p}, a_{k}=a_{q}\right)
$$

where $i$ and $p=1,2, \ldots, M$, and $j$ and $q=1,2, \ldots, N$, and the overall TPM structure is shown in Figure 4.
The representativeness of synthesized cycles is verified by investigating statistically significant criteria. The statistical criteria are determined through generalized linear regression analysis in Lee and Filipi (2011a) and briefly described as follows. Initially, a total number of 27 possible explanatory variables are identified and categorized into velocity related, acceleration related, driving-time and distance-related, and event related variables. Through the assessment of the interrelationship between two variables, one of them is dropped out. Then, 16 variables remain as initial explanatory variables for the regression analysis. Next, generalized linear regression analysis is used to find the least number of significant variables. The analysis includes three assessment steps including $t$-test, normal probability plots of the residuals and histograms of the residuals. The least significant variables are dropped one by one, as long as the reduced equation can represent the response variable with sufficient accuracy. The final regression equations use statistically significant variables to establish bases for subsequent assessments of the representativeness of synthesized driving cycles. The significant explanatory variables are:

- standard deviation of velocity (mph);

- mean positive acceleration $\left(\mathrm{m} / \mathrm{s}^{2}\right)$;

- standard deviation of acceleration $\left(\mathrm{m} / \mathrm{s}^{2}\right)$;

- percentage of driving time under positive acceleration (\%);

- percentage of driving time under negative acceleration (\%);

- mean positive velocity (mph);

- percentage of idle time $(\%)$;

- number of stops/mile ( $1 / \mathrm{mile})$. 


\section{MIDWESTERN US DRIVING CYCLES}

Five cycles are selected to cover the naturalistic driving range and to capture most of naturalistic driving patterns with the driving distance dependency. Figure 5 shows the full set of Urban/Suburban driving cycles typical for the Midwestern US. Each cycle shows different driving patterns. The short distance cycles show more frequent starts and stops, lower velocity and higher acceleration. When the driving distance becomes greater, longer segments with high speed are more frequent.

\subsection{Visual Assessment}

Synthesized driving cycles show clear difference with respect to the driving distance. The shortest cycle (driving distance of 4.87 miles) is the mildest one having the lowest maximum velocity no higher than $53 \mathrm{mph}$ and the most frequent stops. It does not include freeway driving patterns at all over the entire cycles. The longer driving distance, the higher the velocity events start to appear. At the 10.6 mile cycle, velocity profiles become moderately high but still below $65 \mathrm{mph}$. It is well corresponded to the speed limits $(40 \sim 55 \mathrm{mph})$ of local road driving in Southeast Michigan area without severe traffic jam.

Freeway driving patterns become prevalent from medium distance cycles (from 15.5 miles driving distance). At the 25.2 mile cycle, the duration of a continuous freeway driving event is up to 500 seconds and the maximum velocity is up to $80 \mathrm{mph}$ (see Fig. 5d). The medium distance cycles include several local way driving patterns shown in 4.87 mile cycle while showing freeway patterns. In the longest cycle (40.9 miles), the continuous freeway driving event becomes even longer up to 800 seconds. However, the highest speed is mostly maintained below $80 \mathrm{mph}$ owing to the freeway speed limitation (70 mph at freeway, Michigan, US). The cycle includes local way patterns with frequent stops (1 $950 \sim 2500 \mathrm{~s}$ in Fig. 5e) and without frequent stops $(0 \sim 700 \mathrm{~s}$ in Fig. 5e).

\subsection{Trends of Statistical Parameters}

Statistically significant parameters of the proposed cycles have clear trends with respect to driving distance. Tables 1 to 5 show statistical parameters and their comparisons between averaged real-world data and synthetic cycles. The values of parameters from synthetic cycles are well matched to the real-world data. The investigated parameters are:

- mean positive velocity (mph);

- standard deviation of velocity (mph);

- mean positive acceleration $\left(\mathrm{m} / \mathrm{s}^{2}\right)$;

- standard deviation of acceleration $\left(\mathrm{m} / \mathrm{s}^{2}\right)$;

- number of stops per mile (1/mile).
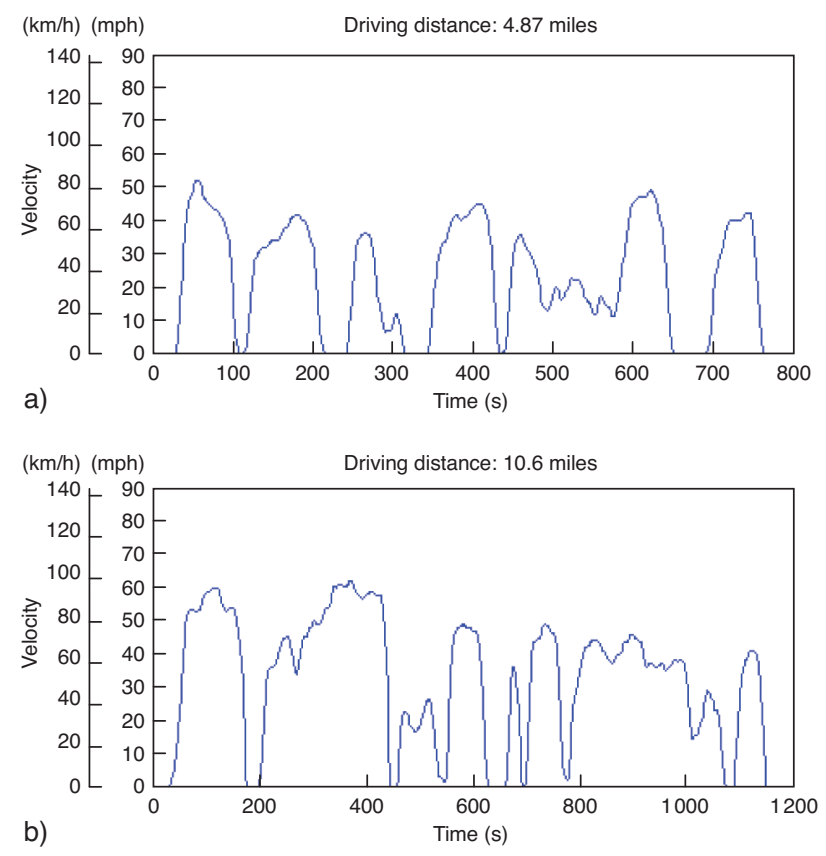

b)
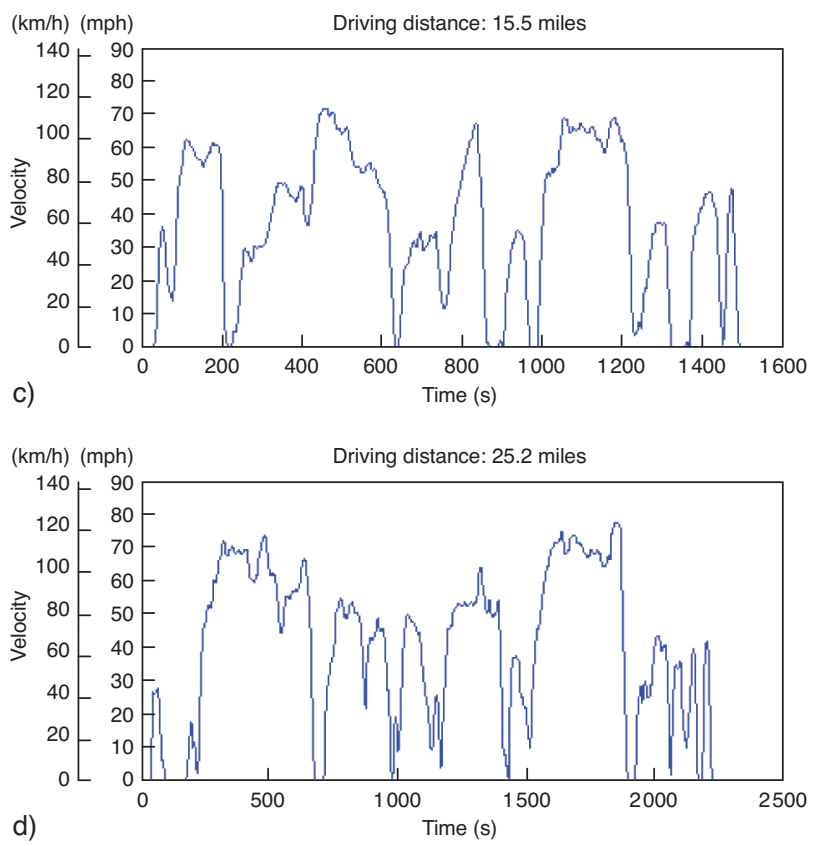

d)

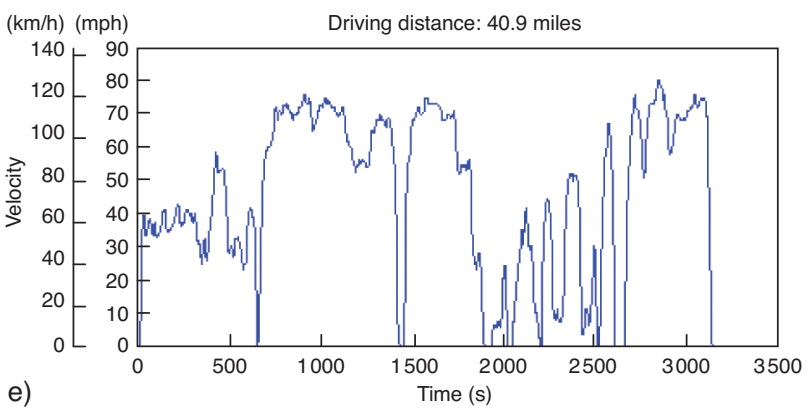

Figure 5

Midwestern US Cycles. 
TABLE 1

Comparison of the statistical parameters of the synthetic driving cycles with 4.87 miles driving distance

\begin{tabular}{l|c|c}
\hline Statistical parameters & Real cycles* & Synthetic cycle \\
\hline Distance (mile) & 4.78 & 4.87 \\
\hline Mean positive velocity $(\mathrm{mph})$ & 27.8 & 27.6 \\
\hline Standard velocity $(\mathrm{mph})$ & 17.3 & 16.9 \\
\hline Mean positive acceleration $\left(\mathrm{m} / \mathrm{s}^{2}\right)$ & 0.99 & 1.02 \\
\hline Standard acceleration $\left(\mathrm{m} / \mathrm{s}^{2}\right)$ & 1.29 & 1.26 \\
\hline Number of stops per mile $(1 / \mathrm{mile})$ & 1.00 & 0.97 \\
\hline
\end{tabular}

* Mean values are presented for real cycles.

TABLE 2

Comparison of the statistical parameters of the synthetic driving cycles with 10.6 miles driving distance

\begin{tabular}{l|c|c}
\hline Statistical parameters & Real cycles* & Synthetic cycle \\
\hline Distance (mile) & 10.6 & 10.6 \\
\hline Mean positive velocity $(\mathrm{mph})$ & 35.5 & 36.5 \\
\hline Standard velocity $(\mathrm{mph})$ & 20.3 & 19.7 \\
\hline Mean positive acceleration $\left(\mathrm{m} / \mathrm{s}^{2}\right)$ & 0.85 & 0.87 \\
\hline Standard acceleration $\left(\mathrm{m} / \mathrm{s}^{2}\right)$ & 1.19 & 1.23 \\
\hline number of stops per mile $(1 / \mathrm{mile})$ & 0.62 & 0.56 \\
\hline
\end{tabular}

* Mean values are presented for real cycles.

TABLE 3

Comparison of the statistical parameters of the synthetic driving cycles with 15.5 miles driving distance

\begin{tabular}{l|c|c}
\hline Statistical parameters & Real cycles* & Synthetic cycle \\
\hline Distance (mile) & 15.4 & 15.5 \\
\hline Mean positive velocity $(\mathrm{mph})$ & 39.9 & 40.3 \\
\hline Standard velocity $(\mathrm{mph})$ & 21.9 & 22.5 \\
\hline Mean positive acceleration $\left(\mathrm{m} / \mathrm{s}^{2}\right)$ & 0.77 & 0.81 \\
\hline Standard acceleration $\left(\mathrm{m} / \mathrm{s}^{2}\right)$ & 1.14 & 1.18 \\
\hline Number of stops per mile $(1 / \mathrm{mile})$ & 0.43 & 0.39 \\
\hline
\end{tabular}

* Mean values are presented for real cycles.

The error criteria of the variables directly related to velocity and acceleration are set to tight $( \pm 5 \%)$.

Trends of the statistical parameters are shown in Figure 6. All presented parameters have clear and smooth trends with respect to driving distance and three of them are shown here. We note that mean positive velocity and mean positive acceleration have an opposite trend. The mean positive velocity is higher, as trip distance is longer. In contrast, the mean positive
TABLE 4

Comparison of the statistical parameters of the synthetic driving cycles with 25.2 miles driving distance

\begin{tabular}{l|c|c}
\hline Statistical parameters & Real cycles* & Synthetic cycle \\
\hline Distance (mile) & 24.9 & 25.2 \\
\hline Mean positive velocity $(\mathrm{mph})$ & 44.9 & 44.3 \\
\hline Standard velocity $(\mathrm{mph})$ & 23.3 & 24.1 \\
\hline Mean positive acceleration $\left(\mathrm{m} / \mathrm{s}^{2}\right)$ & 0.70 & 0.73 \\
\hline Standard acceleration $\left(\mathrm{m} / \mathrm{s}^{2}\right)$ & 1.07 & 1.01 \\
\hline Number of stops per mile $(1 / \mathrm{mile})$ & 0.31 & 0.28 \\
\hline
\end{tabular}

* Mean values are presented for real cycles.

TABLE 5

Comparison of the statistical parameters of the synthetic driving cycles with 40.9 miles driving distance

\begin{tabular}{l|c|c}
\hline Statistical parameters & Real cycles* & Synthetic cycle \\
\hline Distance (mile) & 40.7 & 40.9 \\
\hline Mean positive velocity $(\mathrm{mph})$ & 51.5 & 49.2 \\
\hline Standard velocity (mph) & 24.8 & 24.8 \\
\hline Mean positive acceleration $\left(\mathrm{m} / \mathrm{s}^{2}\right)$ & 0.57 & 0.61 \\
\hline Standard acceleration $\left(\mathrm{m} / \mathrm{s}^{2}\right)$ & 0.93 & 0.87 \\
\hline Number of stops per mile $(1 / \mathrm{mile})$ & 0.19 & 0.17 \\
\hline
\end{tabular}

* Mean values are presented for real cycles.

acceleration decreases along increasing trip distance. The results can be explained as follows: acceleration is directly linked to the velocity change during driving. During short distance trips, frequent starts and stops are prevalent while the driving speed is low. However, long distance trips include a long duration of freeway driving that show high speed cruising without frequent starts and stops. This is well matched to the trend of the number of stops per mile. The number of stops per mile is decreasing from $0.97 \mathrm{stop} / \mathrm{mile}$ at the 4.87 mile cycle to 0.17 at the 40.9 mile cycle.

\subsection{Velocity vs Acceleration Distributions}

Driving patterns with respect to driving distance are assessed by investigating two dimensional plots of velocity versus acceleration distributions. Figure 7 shows that driving patterns are significantly different depending on driving distance. More aggressive acceleration patterns are shown at short distance cycles (see Fig. 7a). At the 4.87 mile cycle, one dominant peak is shown around $30 \mathrm{mph}$ and $1 \mathrm{~m} / \mathrm{s}^{2}$ and it represents local driving. In contrast, long distance cycles show dominant operating events at high speed (above $60 \mathrm{mph}$ ) with moderate acceleration (below $0.5 \mathrm{~m} / \mathrm{s}^{2}$ ) and it represent freeway driving. At the 40.9 mile cycle, the distribution pattern is 
shifted to the higher speed region with a narrowed acceleration range compared to shorter distance cycles as shown in Figure 7c. Medium distance cycles show widely distributed driving patterns as shown in Figure $7 \mathrm{~b}$. The distribution indicates that local way and freeway driving patterns are evenly mixed.
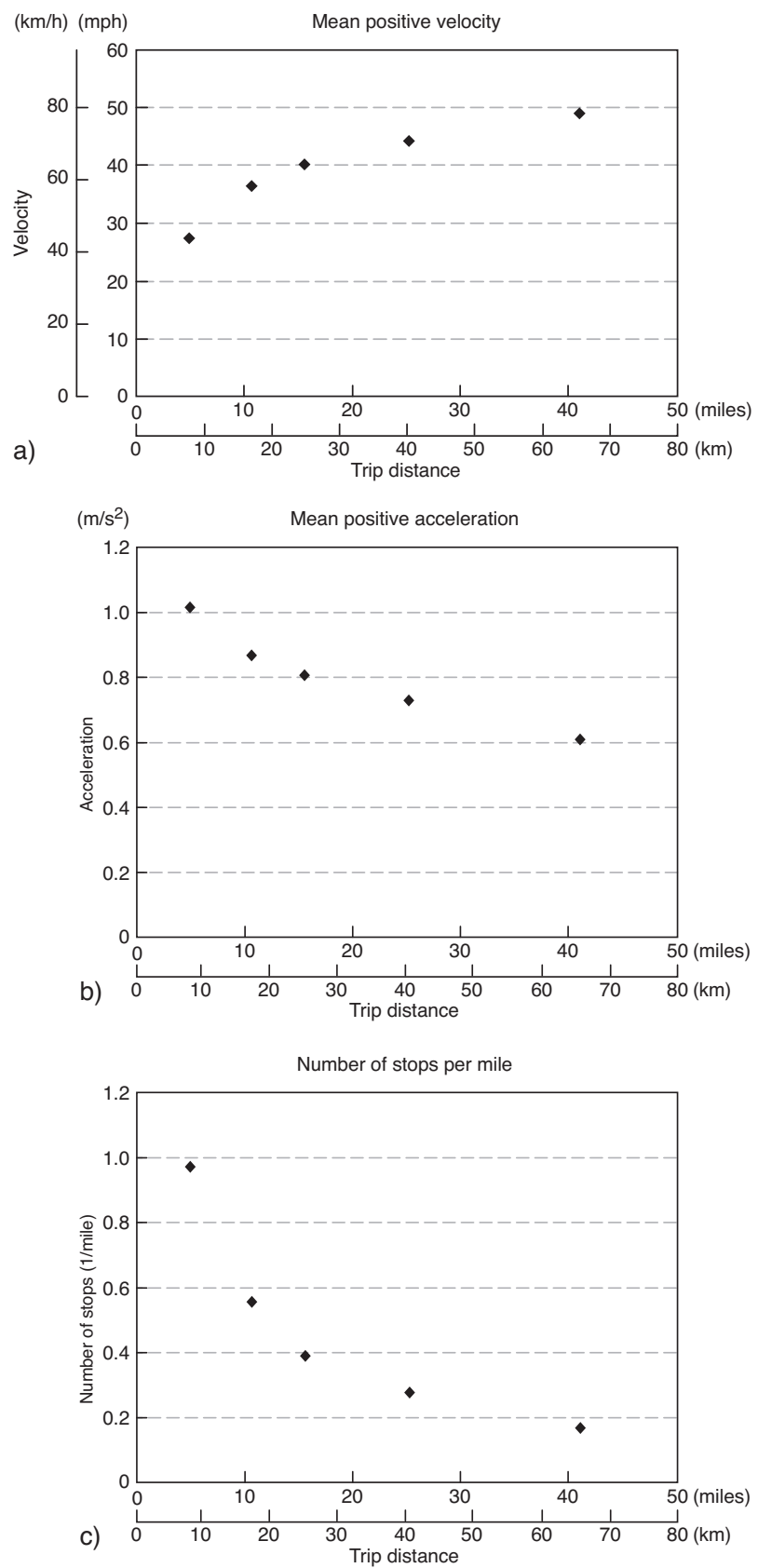

Figure 6

Trends of driving cycle variables with respect to driving distances: a) mean positive velocity, b) mean positive acceleration, c) number of stops per mile.

\section{APPLICATIONS OF REPRESENTATIVE DRIVING CYCLES}

Accurate prediction of PHEV electric load on the grid is important to assess the impact of PHEV penetration on the electric grid and its environmental influence. The electric load prediction requires a large number of driving data and the data could be up to several hundred thousand trips. When detailed vehicle simulations are executed, the prediction accuracy will be significantly improved. However, running detailed simulation with such a large number of data pushes the computational efforts and time beyond manageable limits. Thus, computationally efficient methods are required to deal with a large number of simulation cases.

One way to reduce the computational efforts is avoiding repeating simulations by executing one or a few representative simulations for the case of similar pattern driving cases off-line, then using the off-line simulation results in predicting the PHEV impact on the grid. This concept was proposed by Lee and Filipi (2011b) in a compact representation of PHEV behavior using response surface models. The response surface approach enables prediction of the PHEV electricity demand from the grid and the amount of fuel consumed amount without detailed driving cycle profiles and driving pattern dependency on the trip distance is captured.

The electric energy consumption and the fuel consumption are expressed as functions of driving distance and battery initial State of Charge (SOC). The PHEV responses are predicted by a series PHEV simulation model constructed using Powertrain System Analysis Toolkit (PSAT) developed by Argonne National Laboratory (ANL) and in-house Matlab codes. The model has been validated based on published literature (Carlson et al., 2009; Duoba et al., 2009). Table 6 shows the powertrain model specification for the selected series PHEV. To generate response surfaces over the possible driving distance range and the initial battery SOC, full factorial experiments are design including five representative cycles in the Midwestern cycle set and five additional cycles at each variable.

TABLE 6

Powertrain model specification

\begin{tabular}{l|l}
\hline Component & Specification \\
\hline Engine & $\begin{array}{l}53 \mathrm{~kW} \text {, based on a scaled } 1 \mathrm{~L} \text { Honda Insight } \\
\text { gasoline engine }\end{array}$ \\
\hline Motor/generators & $120 \mathrm{~kW}$ peak UQM power phase PM motor \\
\hline Battery & Li-ion, 6 Ah 75 cell SAFT model scaled to $16 \mathrm{kWh}$ \\
\hline
\end{tabular}

Response surfaces are constructed as shown in Figure 8 and they were originally proposed by Lee and Filipi (2011b). The simulation results were generated to cover wide ranges of trip distances and different battery initial SOCs. To avoid 
a)
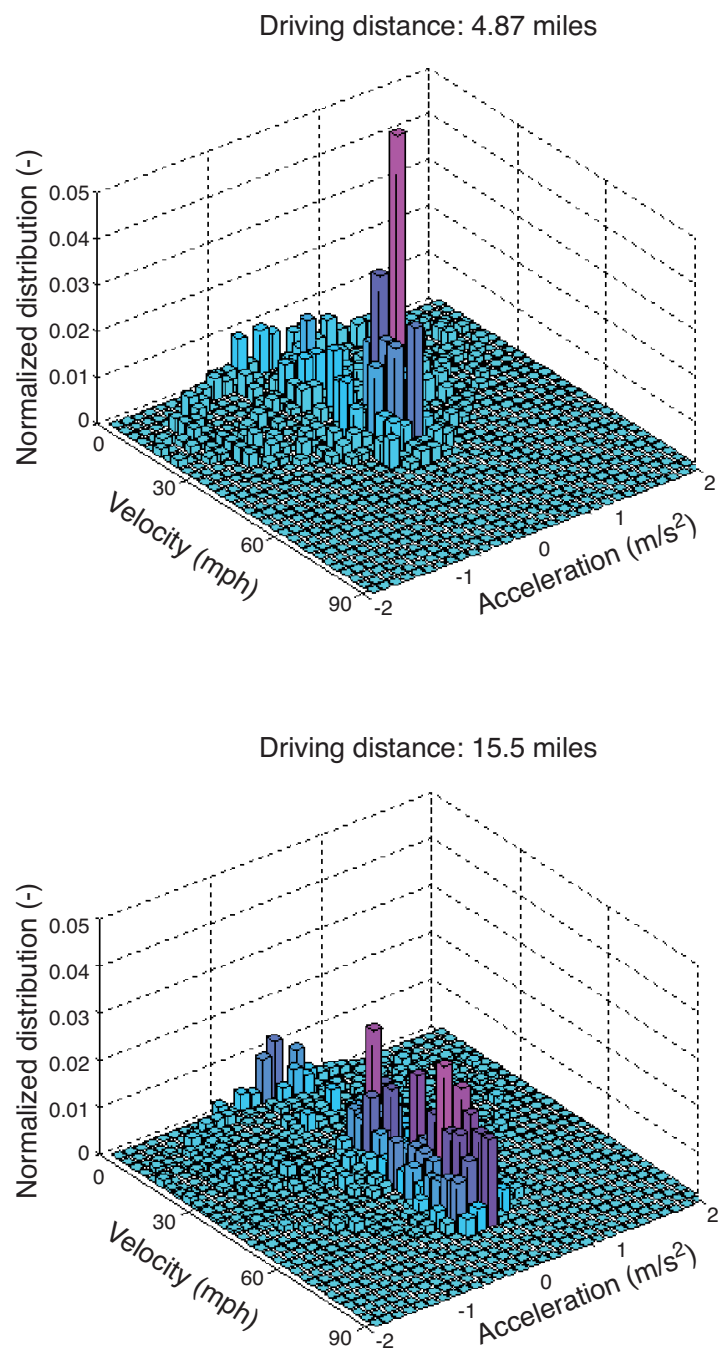

b)

Driving distance: 40.9 miles

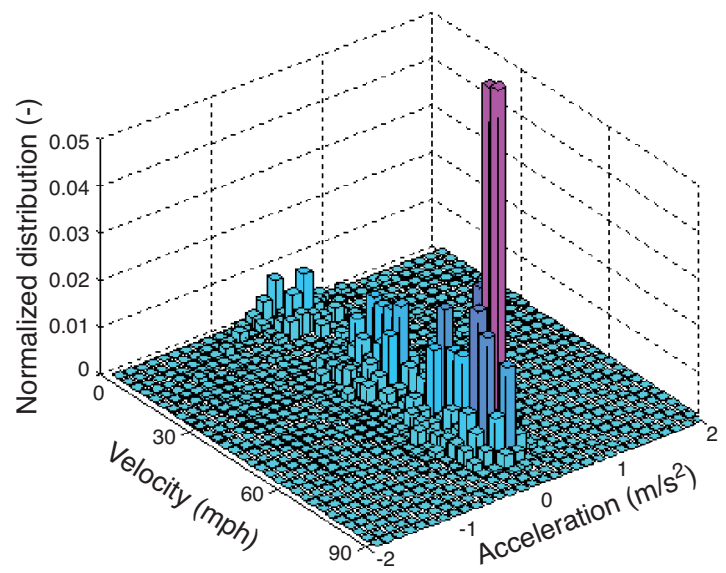

Figure 7

Velocity $v s$ acceleration distributions of Michigan Driving Cycles (MDC): a) 4.78 mile cycle, b) 15.5 mile cycle, c) 40.9 mile cycle.
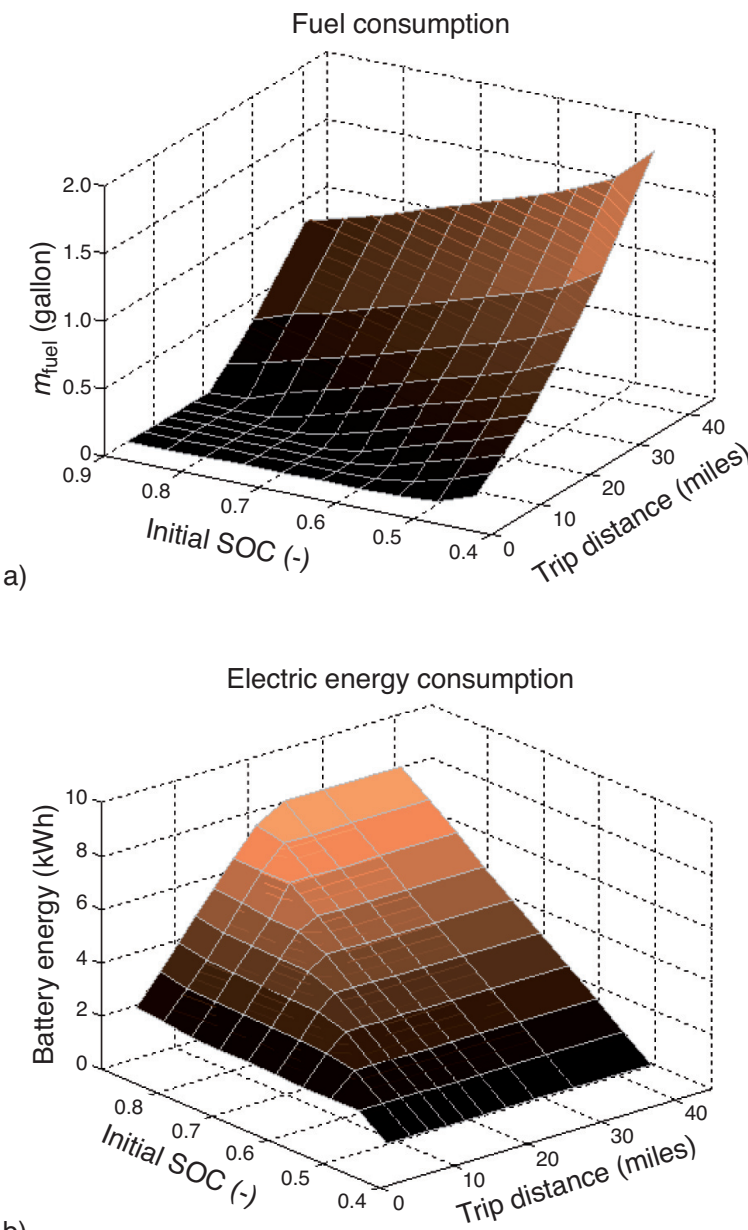

b)

Figure 8

Response surfaces of PHEV behavior computed from the PHEV simulation using representative synthetic real-world cycles: a) the amount of fuel consumption, b) the amount of electric energy consumption.

possible small fluctuations on the response surfaces caused by the random characteristics inherent from the stochastic cycle synthesis process and vehicle supervisory control and to ensure monotonic trend with respect to the initial SOC $\left(\mathrm{SOC}_{\mathrm{ini}}\right)$ and the trip distance, the response surfaces are smoothened through regression analysis.

The response surface models can be used to predict the PHEV impact on the grid under different driving patterns. Figure 9 shows the electricity demand prediction results (Lee and Filipi, 2011b) under two charging scenarios, "charging overnight" and "charging whenever possible" and under three driving patterns:

- naturalistic driving;

- UDDS;

- HWFET. 

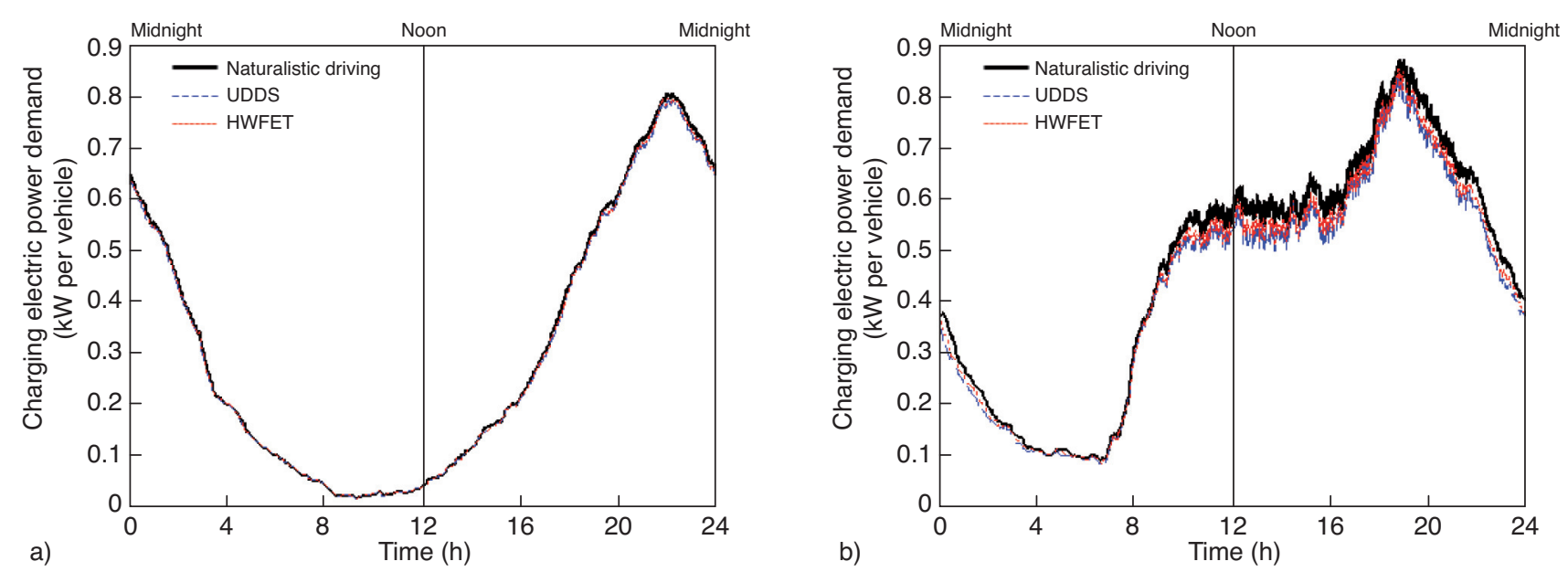

Figure 9

Comparison of the predicted electricity demand at different driving patterns (naturalistic driving, UDDS and HWFET) under: a) "charge overnight" scenario, b) "charge whenever possible" (Lee and Filipi, 2011b).

"Charging overnight" scenario assumes that charging process starts when PHEVs arrive at home and no more trips exist during the day. "Charging whenever possible" scenarios assumes that charging is always possible when a vehicle is parked at any location. The prediction provides quantitative assessment of the electricity demand per vehicle with respect to different charging scenarios. The prediction can be used to predict the total electricity demand of PHEV fleet from the grid by multiplying a penetrated PHEV number.

\section{CONCLUSIONS}

A set of driving cycles is synthesized to represent real-world driving patterns in an urban/suburban area in Midwest US in a compact way. The proposed Midwestern US cycles consist of five one-way trips ranging from 4.87 miles to 40.9 miles. The driving patterns are reconstructed using information extracted from a database of naturalistic driving information in a form of Transfer Probability Matrices (TPMs). The database of naturalistic driving patterns was generated in Southeast Michigan gathered through the Field Operational Tests (FOT) conducted by the University of Michigan Transportation Research Institute (UMTRI). The naturalistic driving data includes 4409 trips covering 830 independent days and temporal distributions of departure and arrival times.

The synthesis procedure is based on the Markov chain to deal with the random characteristics of driving cycles and the subsequent statistical analysis to verify the representativeness. Five synthetic cycles are constructed using data grouped based on the daily driving distance distribution. The synthesized cycles show clear trends of statistical variables, such as mean positive velocity, mean positive acceleration and number of stops per mile. The proposed cycles include both local driving patterns and highway driving patterns and the portion of each pattern changes with respect to the driving distance. An approach for the assessment of the impact of PHEVs on the grid using response surface models is introduced as an example of the application of the Midwestern US cycle set. The Midwestern US cycles will be applicable for Plug-in Hybrid Electric or Electric Vehicle design and control studies, as well as for the assessment of the impact of electrified vehicles on the grid.

\section{ACKNOWLEDGMENTS}

The authors would like to thank Zevi Bareket and Tim Gordon of UMTRI for providing the naturalistic driving data and for their valuable insight pertaining to driver behavior. Funding for this work was provided by the DOE US-China Clean Energy Research Center on Clean Vehicle Collaboration (CERC-CVC) at the University of Michigan.

\section{REFERENCES}

André M. (2004) The ARTEMIS European driving cycles for measuring car pollutant emissions, Sci. Total Environ. 334-335, 73-84.

Carlson R.B., Lohse-Busch H., Duoba M., Shidore N. (2009) Drive cycle fuel consumption variability of plug-in hybrid electric vehicle due to aggressive driving, SAE Technical Paper 2009-01-1335.

Duoba M., Carlson R.B., Bocci D. (2009) Calculating results and performance parameters for PHEVs, SAE Technical Paper 200901-1328. 
Fellah M., Signh G., Rousseau A., Pagerit S., Nam E., Hoffman G. (2009) Impact of real-world drive cycles on PHEV battery requirements, SAE Technical Paper 2009-01-1383.

Kruse R.E., Huls T.A. (1973) Development for the federal urban driving cycle, SAE Technical Paper 730553.

Kwon J., Kim J., Fallas E., Pagerit S., Rousseau A. (2008) Impact of Drive Cycles on PHEV Component Requirements, SAE Technical Paper 2008-01-1337.

LeBlanc D., Sayer J., Winkler C., Ervin R., Bogard S., Devonshire J., Mefford M., Hagan M., Bareket Z., Goodsell R., Gordon T. (2006) Road Departure Crash Warning System Field Operational Test: Methodology and Results, UMTRI-2006-9-2.

Lee T.-K., Filipi Z.S. (2010) Synthesis and validation of representative real-world driving cycles for plug-in hybrid vehicles, IEEE Vehicle Power and Propulsion Conference (VPPC 2010), Lille, France, 1-3 Sept., pp. 1-6.
Lee T.-K., Filipi Z.S. (2011a) Synthesis of real-world driving cycles using stochastic process and statistical methodology, Int. J. Vehicle Design 56, 1, 43-62.

Lee T.-K., Filipi Z.S. (2011b) Response surface modeling approach for the assessment of the PHEV impact on the grid, IEEE Vehicle Power and Propulsion Conference (VPPC 2011), Chicago, IL, 6-9 Sept.

Patil R., Adornato B., Filipi Z.S. (2009) Impact of naturalistic driving patterns on PHEV performance and system design, $S A E$ Technical Paper 2009-01-2715. or distributed for profit or commercial advantage and that copies bear this notice and the full citation on the first page. Copyrights for components of this work owned by others than IFP Energies nouvelles must be honored. Abstracting with credit is permitted. To copy otherwise, to republish, to post on servers, or to redistribute to lists, requires prior specific permission and/or a fee: Request permission from Information Mission, IFP Energies nouvelles, fax. +33147527096 , or revueogst@ifpen.fr. 